\title{
Accelerated drug release and clearance of PEGylated epirubicin liposomes following repeated injections: a new challenge for sequential low-dose chemotherapy
}

\author{
This article was published in the following Dove Press journal: \\ International Journal of Nanomedicine \\ 27 March 2013 \\ Number of times this article has been viewed
}

\author{
Qiang Yang' \\ Yanling $\mathrm{Ma}^{2}$ \\ Yongxue Zhao' \\ Zhennan She' \\ Long Wang' \\ Jie $\mathrm{Li}^{\prime}$ \\ Chunling Wang' \\ Yihui Deng' \\ 'College of Pharmacy, Shenyang \\ Pharmaceutical University, Shenyang, \\ People's Republic of China; ${ }^{2}$ Sichuan \\ Kelun Pharmaceutical Co, Ltd, \\ Chengdu, People's Republic of China
}

Correspondence: Yihui Deng

College of Pharmacy, Shenyang

Pharmaceutical University,

103 Wenhua Road, Shenyang

II0016, Liaoning,

People's Republic of China

Tel +8602423986316

Fax +8602423986316

Email pharmdeng@gmail.com
Background: Sequential low-dose chemotherapy has received great attention for its unique advantages in attenuating multidrug resistance of tumor cells. Nevertheless, it runs the risk of producing new problems associated with the accelerated blood clearance phenomenon, especially with multiple injections of PEGylated liposomes.

Methods: Liposomes were labeled with fluorescent phospholipids of 1,2-dipalmitoyl-snglycero-3-phosphoethanolamine-N-(7-nitro-2-1,3-benzoxadiazol-4-yl) and epirubicin (EPI). The pharmacokinetics profile and biodistribution of the drug and liposome carrier following multiple injections were determined. Meanwhile, the antitumor effect of sequential low-dose chemotherapy was tested. To clarify this unexpected phenomenon, the production of polyethylene glycol (PEG)-specific immunoglobulin M (IgM), drug release, and residual complement activity experiments were conducted in serum.

Results: The first or sequential injections of PEGylated liposomes within a certain dose range induced the rapid clearance of subsequently injected PEGylated liposomal EPI. Of note, the clearance of EPI was two- to three-fold faster than the liposome itself, and a large amount of EPI was released from liposomes in the first 30 minutes in a complement-activation, directdependent manner. The therapeutic efficacy of liposomal EPI following 10 days of sequential injections in S180 tumor-bearing mice of $0.75 \mathrm{mg}$ EPI $/ \mathrm{kg}$ body weight was almost completely abolished between the sixth and tenth day of the sequential injections, even although the subsequently injected doses were doubled. The level of PEG-specific IgM in the blood increased rapidly, with a larger amount of complement being activated while the concentration of EPI in blood and tumor tissue was significantly reduced.

Conclusion: Our investigation implied that the accelerated blood clearance phenomenon and its accompanying rapid leakage and clearance of drug following sequential low-dose injections may reverse the unique pharmacokinetic-toxicity profile of liposomes which deserved our attention. Therefore, a more reasonable treatment regime should be selected to lessen or even eliminate this phenomenon.

Keywords: accelerated blood clearance (ABC) phenomenon, PEGylated liposomes, epirubicin, sequential low-dose injections, complement

\section{Introduction}

Liposomes have been investigated as promising drug carriers over several decades, ${ }^{1,2}$ especially when the emergence of polyethylene glycol (PEG)ylation technology, 3,4 which promotes drugs accumulation within tumor tissues and regions of inflammation, 
capitalizes on the enhanced permeability and retention effect. ${ }^{5,6}$ Nevertheless, recently research found that PEGylated liposomes were cleared rapidly from the blood circulation, following a significantly increased accumulation in the liver and spleen when they were administered twice to the same animal at a certain time interval. ${ }^{7,8}$

The accelerated blood clearance (ABC) phenomenon brings new challenges for clinical applications of PEGylated liposomes since almost all of them require repeated administrations. ${ }^{9}$ Laverman et $\mathrm{al}^{10}$ reported that repeated injections of doxorubicin hydrochloride liposome with $5 \mu \mathrm{mol}$ phospholipids/kg body weight in rats did not alter the pharmacokinetics of the subsequent injections of indium-111 labeled PEGylated liposomal doxorubicin. Consistent with the above results, Ishida et $\mathrm{al}^{11}$ demonstrated that the first injection of liposomal doxorubicin failed to produce increased clearance of the second dose. Now, a more reasonable explanation for PEGylated doxorubicin liposomes failure to induce the $\mathrm{ABC}$ phenomenon is that the first injected liposomes are partly taken up by the spleen, and the drug released from the liposomes inhibits the proliferation of splenic marginal zone B cells which reduces the secretion of anti-PEG immunoglobulin $\mathrm{M}(\operatorname{IgM}) .{ }^{12}$

It is noteworthy that the most common method for studying the ABC phenomenon is to investigate the pharmacokinetic behavior and tissue distribution of carriers after double or multiple injections. ${ }^{10,11,13}$ Nevertheless, for the drug delivery system, the carrier only behaves as a transport, and the definitive changes in the pharmacokinetic behavior of drugs in vivo are the most fundamental objective. Recently, we prepared the PEGylated liposomes loaded with topotecan to study the ABC phenomenon of the drug after repeated injections in rats. ${ }^{14}$ The results showed that the $\mathrm{ABC}$ phenomenon was attenuated with the increase of the first dose of empty and topotecan liposomes. However, this phenomenon still did not disappear even though the doses were $20 \mu \mathrm{mol}$ phospholipids/ $/ \mathrm{kg}$ and $1.6 \mathrm{mg}$ topotecan $/ \mathrm{kg}$, respectively. These findings prompted us to do more detailed research of the $\mathrm{ABC}$ phenomenon of cytotoxic drugs and PEGylated liposomes.

To track the in vivo behavior of the carrier, liposomes loaded or labeled with radionuclides such as indium-111, technetium-99m, and gallium-67 were introduced in the 1970s. ${ }^{15}$ Although radio labeling is the conventional method to monitor the in vivo pharmacokinetic behavior of liposomes, it has a radiation hazard. Radioactive contamination is difficult to deal with and the conventional laboratory without radiation protection cannot carry out the operation safely. 1,2-dipalmitoyl-sn-glycero-3-phosphoethanolamine$\mathrm{N}$-(7-nitro-2-1,3-benzoxadiazol-4-yl) (NBD-PE) is one of the phospholipid derivatives which has wide applications in studying the membrane fusion and cellular uptake of liposomes. ${ }^{16,17}$ It is easy to mix with lipids and stably exists in the liposome bilayer. Therefore, in order to facilitate the process of preparation and detection of liposomes, fluorescent NBD-PE phospholipids were used to serve as a marker to investigate the in vivo fate of the liposome carrier in the present study.

In recent years, a new treatment regimen of sequential low-dose chemotherapy, so-called "metronomic" chemotherapy, has been widely used, especially when combined with radiation therapy and antiangiogenic agents. ${ }^{18-20}$ Compared with conventional chemotherapy involving infusion of maximum tolerated doses, this method significantly reduces the toxic side effects and, furthermore, it reduces the risk of multidrug resistance by tumor cells. ${ }^{21}$ However, the appearance of the ABC phenomenon may become a potential threat to this regimen. Moreover, the occurrence of the $\mathrm{ABC}$ is accompanied by rapid activation of the complement system, and the interaction of the complement with subsequently injected liposomes may promote the leakage of encapsulated drugs via the formation of a membrane attack complex..$^{22,23}$ The leakage and clearance of drugs may hamper the antitumor activity of low-dose sequential injections. If this happens, it will obstruct the development of sequential low-dose injections of PEGylated liposomes.

Therefore, in the present study, the initial purpose was to label the carrier with fluorescent probe NBD-PE and investigate the effect of repeated and sequential injections of PEGylated liposomes on the degree of the ABC phenomenon, especially the different in vivo fates of the liposomes and epirubicin (EPI). A second, but more important, purpose was to evaluate whether the leakage and clearance of EPI could hamper the antitumor activity of low-dose sequential injections. This article investigated the pharmacokinetics and pharmacodynamics of EPI during the ABC phenomenon in detail, and our findings may have great significance in guiding the clinical application of PEGylated liposomes, especially when administrated in the form of low-dose injections.

\section{Materials and methods}

Hydrogenated soy phosphatidylcholine (HSPC) was purchased from Lucas Meyer (Düsseldorf, Germany). EPI HCl (EPI, purity $99.0 \%$ by high-performance liquid chromatography) was purchased from Olympic Star Pharmaceutical Co, Ltd (Shenzhen, People's Republic of China). Cholesterol was 
obtained from Nanjing Xinbai Pharmaceutical Co, Ltd (Nanjing, People's Republic of China). 1,2-distearoyl-snglycero-3-phosphoethanolamine-N-[methoxy (polyethylene glycol)-2000] ( $\mathrm{mPEG}_{2000}$-DSPE) was purchased from Genzyme Corporation (Cambridge, MA, USA). NBD-PE was purchased from Avanti Polar Lipids (Alabaster, Alabama, USA). ZB-1 cation and ZB-2 anion exchange fibers were purchased from Guilin Zhenghan Technology Development Co, Ltd (Guilin, People's Republic of China). Sheep red blood cells and hemolysin (rabbit anti-sheep red blood cell antibody) were purchased from South Reagent Factory (Yuhuan, People's Republic of China). All other chemicals were of analytical grade.

\section{Animals}

Male Wistar rats (200-240 g) and male KM mice (18-22 g) were purchased from the Laboratory Animal Center of Shenyang Pharmaceutical University (Shenyang, People's Republic of China). All rats and mice had free access to food and water. All care and animal experiments were carried out in accordance with the guidelines of the Animal Welfare Committee of Shenyang Pharmaceutical University.

\section{Preparation of liposomes}

The PEGylated empty liposomes were prepared according to a previous report. ${ }^{24}$ Briefly, a certain amount of ethanol was added to solubilize the mixture of HSPC, cholesterol, and $\mathrm{mPEG}_{2000}$-DSPE (56.4:38.4:5.2, molar ratio), and the residual solvent was removed at $65^{\circ} \mathrm{C}$. Then, the dry lipid film was hydrated at $65^{\circ} \mathrm{C}$ for 20 minutes with ammonium sulfate solution $\left(200 \mathrm{mM}\left(\mathrm{NH}_{4}\right)_{2} \mathrm{SO}_{4}, \mathrm{pH}\right.$ 5.5). The final lipid concentration was $50 \mathrm{mg} / \mathrm{mL}$. To obtain uniform large unilamellar vesicles, the suspensions were passed through 0.8 , 0.45 , and $0.22 \mu \mathrm{m}$ filter membranes after sonication with a laboratory ultrasonic cell pulverizer (JY92-II; Ningbo Scientz Biotechnology Co, Ltd, Zhejiang, People's Republic of China). The mean diameters and zeta potentials of the liposomes were determined using a NICOMP 380 HPL submicron particle analyzer (Particle Sizing Systems, Port Richey, FL, USA). In addition, a Vapro ${ }^{\circledR} 5520$ vapor pressure osmometer (Wescor
Inc, Logan, UT, USA) was used to measure the osmotic pressure of liposomes.

To investigate the pharmacokinetics and biodistribution of the liposome itself, the fluorescently labeled liposomes were prepared with the same process as above. The lipid composition was as follows: HSPC, cholesterol, $\mathrm{mPEG}_{2000}-\mathrm{DSPE}$, and NBD-PE (56.4:38.4:5.2:0.56, molar ratio).

\section{The establishment of an ion gradient and encapsulation of EPI}

In the present study, EPI was loaded into liposomes using an ammonium sulfate gradient. ${ }^{25}$ The external buffer of the liposomes was exchanged by passage through a ZB-1 cation and ZB-2 anion exchange fiber prepared column that was equilibrated with $300 \mathrm{mM}$ sucrose, and the liposomal dispersion and EPI solution $(4 \mathrm{mg} / \mathrm{mL})$ were mixed at a drug to lipid ratio of 1:10 (wt/wt), then incubated for 20 minutes at $60^{\circ} \mathrm{C}$. After loading, untrapped EPI was removed with a cation exchange fiber column and the entrapment efficacy was assessed photometrically at $480 \mathrm{~nm}$ (UV1801 UV/VIS Spectrophotometer; Beijing Rayleigh Analytical Instrument Co, Ltd, Beijing, People's Republic of China) after solubilization of liposomes with $90 \%(\mathrm{v} / \mathrm{v})$ isopropyl alcohol containing $0.75 \mathrm{~mol} / \mathrm{L} \mathrm{HCl}$. The concentration of phospholipids was measured by colorimetric assay. ${ }^{26}$ Furthermore, the general parameters were also determined as described above, and the detailed information is shown in Table 1.

\section{Pharmacokinetics and biodistribution of PEGylated liposomes}

Male Wistar rats were randomly divided into ten groups. The rats were initially injected via the tail vein with PEGylated liposomes (without EPI) at a dose of 1, 5, 10, 15, or $50 \mu \mathrm{mol}$ phospholipids $/ \mathrm{kg}$, and the injection protocols with EPI were the same as above except for $50 \mu \mathrm{mol}$ phospholipids $/ \mathrm{kg}$. The detailed injection scheme is presented in Table 2. Control animals received an injection of $5 \%$ glucose instead of liposomes. The interval between the two injections was 7 days, and the serum samples were collected 30 minutes before the second injection. For the second injection, EPI encapsulated and NBD-PE-labeled test liposomes were injected intravenously

Table I The general parameters of liposomes

\begin{tabular}{|c|c|c|c|c|c|}
\hline Formulation & $\begin{array}{l}\text { Vesicle } \\
\text { size/nm }\end{array}$ & PI & $\begin{array}{l}\text { Zeta potentials/ } \\
\text { mV }\end{array}$ & $\begin{array}{l}\text { Osmotic pressure/ } \\
\mathrm{mOsmol} / \mathrm{kg}\end{array}$ & $\begin{array}{l}\text { Entrapment } \\
\text { efficacy } / \%\end{array}$ \\
\hline Empty liposome & $110.3 \pm 5.4$ & $0.16 \pm 0.01$ & $-22.4 \pm 0.4$ & $249 \pm 2.6$ & l \\
\hline Fluorescently labeled empty liposome & $1 \mid 4.7 \pm 4.9$ & $0.18 \pm 0.02$ & $-26.1 \pm 0.7$ & $253 \pm 3.1$ & 1 \\
\hline EPI-encapsulated liposome & $112.6 \pm 6.7$ & $0.19 \pm 0.02$ & $-21.1 \pm 0.6$ & $272 \pm 3.9$ & $98.6 \pm 0.8$ \\
\hline
\end{tabular}

Abbreviations: EPI, epirubicin; PI, polydispersion index. 
Table 2 The injection scheme of the PEGylated liposomes

\begin{tabular}{|c|c|c|}
\hline Group & First injection & Second injection \\
\hline A & $5 \%$ glucose injection & $\begin{array}{l}5 \mu \mathrm{mol} \\
\text { phospholipids } / \mathrm{kg} \\
\text { EPI liposome or } \\
5 \mu \mathrm{mol} \\
\text { phospholipids/kg } \\
\text { fluorescently labeled } \\
\text { empty liposome }\end{array}$ \\
\hline B & $\begin{array}{l}\text { I } \mu \text { mol phospholipids/kg } \\
\text { empty liposome }\end{array}$ & \\
\hline C & $\begin{array}{l}5 \mu \mathrm{mol} \text { phospholipids/kg empty } \\
\text { liposome }\end{array}$ & \\
\hline $\mathrm{D}$ & $\begin{array}{l}\text { I0 } \mu \mathrm{mol} \text { phospholipids } / \mathrm{kg} \\
\text { empty liposome }\end{array}$ & \\
\hline$E$ & $\begin{array}{l}\text { I } 5 \mu \mathrm{mol} \text { phospholipids } / \mathrm{kg} \\
\text { empty liposome }\end{array}$ & \\
\hline $\mathrm{F}$ & $\begin{array}{l}50 \mu \mathrm{mol} \text { phospholipids } / \mathrm{kg} \\
\text { empty liposome }\end{array}$ & \\
\hline G & $\begin{array}{l}\text { I } \mu \mathrm{mol} \text { phospholipids } / \mathrm{kg} \\
\text { epiribicin liposome (correspond } \\
\text { to } 0.08 \mathrm{mg} \mathrm{EPI} / \mathrm{kg} \text { ) }\end{array}$ & \\
\hline $\mathrm{H}$ & $\begin{array}{l}5 \mu \mathrm{mol} \text { phospholipids } / \mathrm{kg} \\
\text { epiribicin liposome (correspond } \\
\text { to } 0.4 \mathrm{mg} \mathrm{EPI} / \mathrm{kg} \text { ) }\end{array}$ & \\
\hline I & $\begin{array}{l}\text { I0 } \mu \mathrm{mol} \text { phospholipids } / \mathrm{kg} \\
\text { epiribicin liposome (correspond } \\
\text { to } 0.8 \mathrm{mg} \mathrm{EPI} / \mathrm{kg} \text { ) }\end{array}$ & \\
\hline J & $\begin{array}{l}\text { I5 } \mu \mathrm{mol} \text { phospholipids } / \mathrm{kg} \\
\text { epiribicin liposome (correspond } \\
\text { to } 1.2 \mathrm{mg} \mathrm{EPI} / \mathrm{kg} \text { ) }\end{array}$ & \\
\hline
\end{tabular}

Abbreviation: EPI, epirubicin; PEG, polyethylene glycol.

at a dose of $5 \mu \mathrm{mol}$ phospholipids $/ \mathrm{kg}$ separately. At 0.083 , $0.25,0.5,1,2$, and 4 hours following intravenous injection, $500 \mu \mathrm{L}$ of blood samples were obtained by eye puncture. After withdrawing the last blood sample at 4 hours, the livers and spleens were excised, rinsed in ice-cold normal saline, and snap frozen. Blood samples were centrifuged at $4000 \mathrm{rpm}$ for 10 minutes to separate the plasma. The plasma and tissue samples were stored at $-20^{\circ} \mathrm{C}$ until assayed.

In order to evaluate the effect of sequential low-dose chemotherapy on the pharmacokinetics and biodistribution of PEGylated liposomes, another ten groups of rats were used. The rats were sequentially injected with $0.75 \mathrm{mg}$ liposomal $\mathrm{EPI} / \mathrm{kg}$, and from group one to ten, the corresponding days of sequential injections were one to ten, respectively. Thirty minutes before the last injection, the serum samples were collected. After the last injection of each group, blood, liver, and spleen samples were obtained by the same method as above.

\section{Analytical procedure}

The concentrations of EPI and NBD-PE in plasma and tissue samples were determined using a spectrofluorometric method. Before analysis, the tissues were first homogenized using a Tissue Tearor equipped with an $80 \mathrm{~mm}$ probe (IKA WORKS GUANGZHOU, Guangzhou, People's Republic of China). Before EPI and NBD-PE analysis, the samples were treated as follows. To $100 \mu \mathrm{L}$ plasma and $200 \mu \mathrm{L}$ tissue homogenate, 3.9 and $3.8 \mathrm{~mL}$ extraction solution (methanol/water, $50 / 50$ containing $0.3 \mathrm{M} \mathrm{HCl}$ ) was added, the mixture was then vortexed for 30 seconds, and the supernatant was collected by centrifuging the mixture at $10,000 \mathrm{rpm}$ for 10 minutes. The supernatants were assayed using a 650-60 fluorescence spectrophotometer (Hitachi Ltd, Tokyo, Japan), and the emission wavelength/excitation wavelength of EPI and NBD-PE were $502 / 562 \mathrm{~nm}$ and $488 / 540 \mathrm{~nm}$, respectively.

\section{Quantification of IgM}

As previously reported, ${ }^{27,28}$ anti-PEG IgM arising from the initial injections of liposomes was responsible for the induction of the $\mathrm{ABC}$ phenomenon. It has been used as an indicator for evaluating the extent of enhanced blood clearance..$^{29}$ In order to obtain a better explanation of the results, the determination of anti-PEG IgM in rats and mice was conducted according to a previously published procedure with a slight modification. ${ }^{29}$ Quantification of $\operatorname{IgM}$ was performed using a Rat and Mouse IgM enzyme-linked immunosorbent assay Quantitation Kit (Beijing Biosynthesis Biotechnology Co, Ltd, Beijing, People's Republic of China). In brief, $50 \mu \mathrm{L} \mathrm{mPEG}_{2000}$-DSPE ethanol solution $(0.2 \mathrm{mmol} / \mathrm{L})$ was added to each well of a $96-$ well microplate (Corning Incorporated, Corning, NY, USA) and thoroughly air dried. Then, $100 \mu \mathrm{L}$ of the blocking solution $(50 \mathrm{mM}$ Tris, $1 \%$ bovine serum albumin, $\mathrm{pH} 8.0$ ) was added to each well, and the plate was incubated for 30 minutes. After incubation, the wells were washed five times with Tris-buffered saline containing $0.05 \%$ Tween 20 . Then $100 \mu \mathrm{L}$ samples of serum in the sample diluent ( $1 \%$ bovine serum albumin, $0.05 \%$ Tween $20,50 \mathrm{mM}$ Tris, $\mathrm{pH} 8.0$ ) were added to the wells, followed by incubation for 30 minutes and washing five times as described above. Horseradish peroxidase (HRP)-conjugated antibody $(100 \mu \mathrm{L}, 1 \mu \mathrm{g} / \mathrm{mL}$, goat antirat IgM-HRP conjugate, and goat anti-mouse IgM-HRP conjugate) was added to each well. After incubation for 30 minutes, the wells were washed five times as described above. Staining was carried out by the addition of $100 \mu \mathrm{L}$ o-phenylene diamine (1 mg/mL; Sigma-Aldrich, St Louis, MO, USA). After incubation for 5 minutes, the reaction was stopped by adding $100 \mu \mathrm{L} 2 \mathrm{~N} \mathrm{H}_{2} \mathrm{SO}_{4}$, and the absorbance was measured at $490 \mathrm{~nm}$ using a microplate reader (BioRad Laboratories Ltd, Hercules, CA, USA). All incubations were performed at $37^{\circ} \mathrm{C}$. 


\section{In vitro release rate of EPI in serum}

PEGylated EPI liposomes were used to investigate the leakage of EPI, and untrapped EPI was removed with a cation exchange fiber column equilibrated with $300 \mathrm{mM}$ sucrose. Briefly, $100 \mu \mathrm{L}$ liposomes $(2.5 \mu \mathrm{mol}$ phospholipids $/ \mathrm{mL})$ were added to $900 \mu \mathrm{L}$ serums, and the mixture was incubated at $37^{\circ} \mathrm{C}$. Serum was obtained from rats that had been pretreated with glucose solution, empty, and EPI-encapsulated liposomes. The release of EPI from liposomes was measured as follows: at $0.083,0.25,0.5,1,2$, and 4 hours, a $50 \mu \mathrm{L}$ serum fraction was passed through a cation fiber column to separate the liposomeencapsulated EPI from the free EPI as previously reported. ${ }^{30}$ The spectrofluorometric method was used to determine the encapsulation efficiency (EE) of the samples.

The percentage EPI release $\left(\mathrm{R}_{\mathrm{EPI}}\right)$ was calculated using the following equation:

$$
\mathrm{R}_{\mathrm{EPI}} \%=100 \%-\mathrm{EE} \% \text {. }
$$

\section{The determination of residual complement activity in serum}

Fifty percent hemolytic complement activity (CH50) was determined to characterize the residual serum complement activity using a slightly modified procedure based on the published method. ${ }^{11}$ Briefly, $180 \mu \mathrm{L}$ of serum obtained from rats on the seventh day after intravenous injection of $5 \%$ glucose or PEGylated liposomes was incubated with $20 \mu \mathrm{L}$ of PEGylated liposomes $(2.5 \mu \mathrm{mol}$ phospholipids $/ \mathrm{mL})$ at $37^{\circ} \mathrm{C}$ for 15 minutes. The mixture was 1:20, 1:10, or 1:5 diluted with phosphate buffered solution $\left(3.98 \mathrm{mM} \mathrm{Na}_{2} \mathrm{HPO}_{4} \cdot 12 \mathrm{H}_{2} \mathrm{O}\right.$, $\left.1 \mathrm{mM} \mathrm{KH}_{2} \mathrm{PO}_{4}, 145.55 \mathrm{mM} \mathrm{NaCl}, 0.83 \mathrm{mM} \mathrm{MgSO}_{4}\right)$. Then a certain amount of diluted serum was added to the centrifuge tube, adding the phosphate buffered solution to the total volume of 1.5 L. Lastly, $1.0 \mathrm{~mL}$ of sensitization red blood cells (SRBCs) was mixed with the above contents mixture of the centrifuge tube and incubated at $37^{\circ} \mathrm{C}$ for 1 hour. Hemolysisfree SRBCs were pelleted by centrifugation at $3000 \mathrm{rpm}$ for 30 minutes, and the supernatant was quantified photometrically at $541 \mathrm{~nm}$ (UV-1801 UV/VIS Spectrophotometer). The $\mathrm{CH} 50$ was calculated in accordance with instructions and the residual complement activity was assayed by dividing the CH50 of samples with the CH50 of blank serum.

\section{In vivo anti-tumor activity of EPI liposomes after sequential injections at a low dose}

To investigate the effects of EPI leakage and clearance during the $\mathrm{ABC}$ phenomenon on the anti-tumor activity of EPI liposomes, an S180 tumor model was prepared by subcutane- ously injecting $2 \times 10^{6} \mathrm{~S} 180$ tumor cells in $0.2 \mathrm{~mL}$ saline into the right armpit of mice, then randomly dividing the animals into five groups. The third day after transplantation, S180 tumor-bearing mice were injected via the tail vein with an EPI dose of $0.75 \mathrm{mg}$ liposomal EPI $/ \mathrm{kg}$ (Low EPI-L), $1.5 \mathrm{mg}$ liposomal EPI/kg (High EPI-L), and $0.75 \mathrm{mg}$ free EPI $/ \mathrm{kg}$ (free-EPI), or the same volumes of 5\% glucose (Control), once daily for 10 days. The fifth group was injected with $0.75 \mathrm{mg}$ liposomal EPI $/ \mathrm{kg}$ for the first 5 days, and then the dose was increased to $1.5 \mathrm{mg}$ liposomal EPI/ $\mathrm{kg}$ (Low plus high EPI-L). The tumor volume was calculated using the equation:

$$
0.5 \times \text { length } \times \text { width }^{2} .
$$

For statistical analysis, the tumor sizes were determined every day. On the sixth and tenth day, serum samples were collected 30 minutes before administration and 4 hours after injection, five mice were sacrificed, and plasma and tissue samples were collected. Then, anti-PEG IgM was determined as described in the Methods section and the concentrations of EPI in blood and tissue were measured.

\section{Statistical analysis}

All data are expressed as mean \pm standard deviation. Statistical comparisons were performed using Student's $t$-test with SPSS software (v 17.0; IBM Corporation, Armonk, NY, USA). $P$-values lower than 0.05 were considered statistically significant.

\section{Results \\ Effect of first injection of empty and EPI encapsulated PEGylated liposomes on the in vivo clearance of NBD-PE and EPI labeled PEGylated liposomes}

To investigate the impact of the first injection of phospholipids and EPI doses on inducing the $\mathrm{ABC}$ phenomenon, we treated rats with empty and EPI-encapsulated liposomes in accordance with Table 2. The results shown in Figure 1A were similar to those reported in previous studies, ${ }^{31}$ and the control group had a long circulation time. On increasing the phospholipids and EPI doses of the first injection, the extent of the $\mathrm{ABC}$ phenomenon on the second injection of EPI liposomes was reduced. The enhanced clearance of the second dose was abolished when the first dose reached $50 \mu \mathrm{mol}$ phospholipids $/ \mathrm{kg}$ and $1.2 \mathrm{mg}$ EPI $/ \mathrm{kg}$. However, from the results of Figure 1B we can see that the $\mathrm{ABC}$ phenomenon of PEGylated liposomes was obviously attenuated, especially in the first 30 minutes. This means that the clearance of 

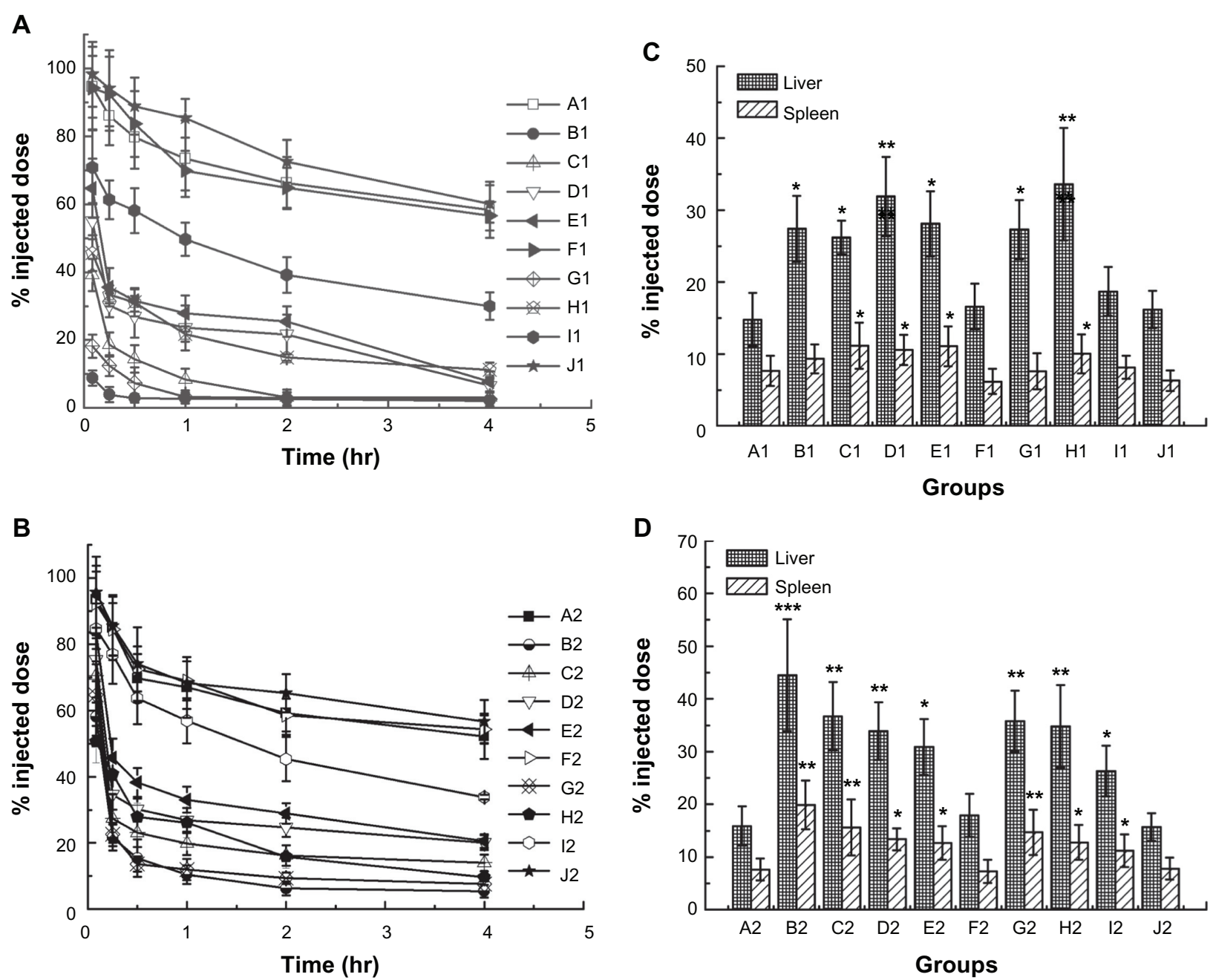

Figure I (A) Blood clearance of epirubicin. From groups A I to JI represents pre-dosing with $5 \%$ glucose injection, I, 5, I0, I5, or $50 \mu$ mol phospholipids/kg empty liposomes and I, 5, 10, or $15 \mu \mathrm{mol}$ phospholipids/kg epirubicin liposomes, respectively. (B) Blood clearance of NBD-PE labeled liposomes. (C) Hepatic and splenic accumulation of epirubicin, 4 hours after intravenous injection of the test dose. (D) Hepatic and splenic accumulation of NBD-PE labeled liposomes. Groups A2 to J2 represents the same protocol of pre-dosing as (A).

Notes: Data are shown as mean $\pm S D, n=3$. P-values apply to differences between the control and treated rats. ${ }^{*} P<0.05 ; * * P<0.01 ; * * * P<0.001$.

Abbreviation: NBD-PE, I,2-dipalmitoyl-sn-glycero-3-phosphoethanolamine-N-(7-nitro-2-I,3-benzoxadiazol-4-yl). SD, standard deviation.

EPI and liposomes may be different in vivo upon repeated injections.

Another obvious change in the $\mathrm{ABC}$ phenomenon was the distribution of carriers or drugs. As shown in Figure 1C, the control rats exhibited low levels of accumulation of the injected dose in the liver and spleen at 4 hours after injection. However, the enhanced liver and spleen accumulation of the second dose were significantly different between the liposome treated groups and the control group. It is worth noting that in groups $\mathrm{B}, \mathrm{G}$, and $\mathrm{H}$ the amount of EPI accumulated in the liver was only doubled even though a very marked $A B C$ effect was observed. It was far lower than the results shown in Figure 1D for the amounts of fluorescent-labeled empty PEGylated liposomes, which tripled in the liver.

\section{The $A B C$ phenomenon induced by sequential low-dose chemotherapy}

The above results indicated that a low-dose first injection would lead to the rapid clearance of the second injected liposomes. However, whether the sequential low-dose injections could induce the $\mathrm{ABC}$ phenomenon is unknown. To illustrate the relationship between the pharmacokinetics and biodistribution behavior of the last administration of PEGylated liposomes and the days of sequential injections, a regime of sequential lowdose chemotherapy was carried out in rats. As shown in Figure 2A, a comparatively increased blood clearance was observed after 5 days of repeated injections. The most serious $\mathrm{ABC}$ effect was induced during 6 to 10 days of injections, and followed by increased hepatic and splenic 

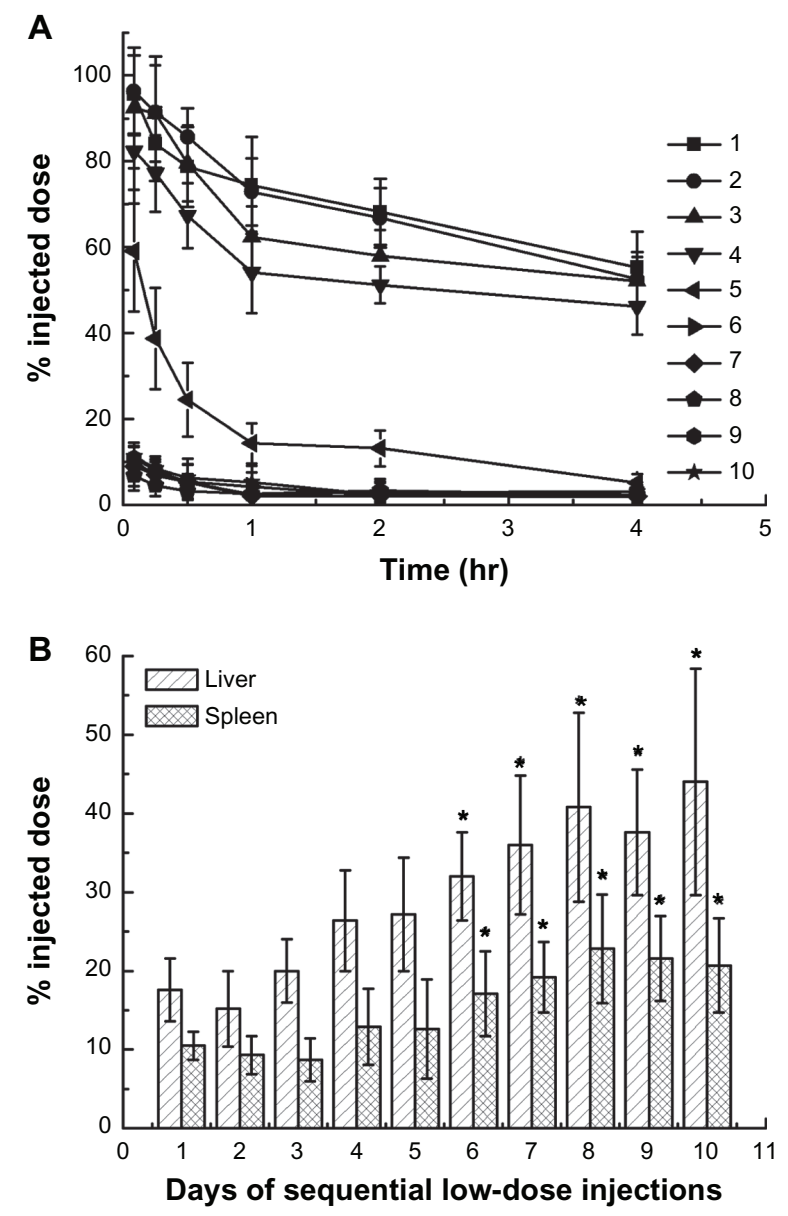

Figure 2 Effect of days of sequential injections of epirubicin-encapsulated PEGylated liposomes on the pharmacokinetics and biodistribution of the last dose. (A) Blood clearance. One to 10 represents the number of days of sequential injection with $0.75 \mathrm{mg}$ liposomal epirubicin/kg. (B) Hepatic and splenic accumulation, 4 hours after intravenous injection of the last dose.

Notes: Data are shown as mean $\pm \mathrm{SD}, \mathrm{n}=3$. $P$-values apply to differences between the control and treated rats. $* P<0.05$.

Abbreviation: PEG, polyethylene glycol; SD, standard deviation.

accumulation of injected low-dose EPI encapsulated liposomes (see Figure 2B).

\section{Determination of the production of anti-PEG IgM}

Anti-PEG IgM is responsible for the marked alteration of pharmacokinetic behavior of subsequent doses. ${ }^{28}$ Therefore, we investigated the effect of liposomes, with or without EPI, on IgM production. The serum was prepared from rats on day 7 after the first injection or the last day of repeated injections. As shown in Figure 3A, at the same dose of phospholipids, the encapsulation of EPI partly inhibited the secretion of anti-PEG IgM. This may mainly be because the splenic marginal zone B cells were partly inhibited or even destroyed by the first injection of cytotoxic drugs. However,
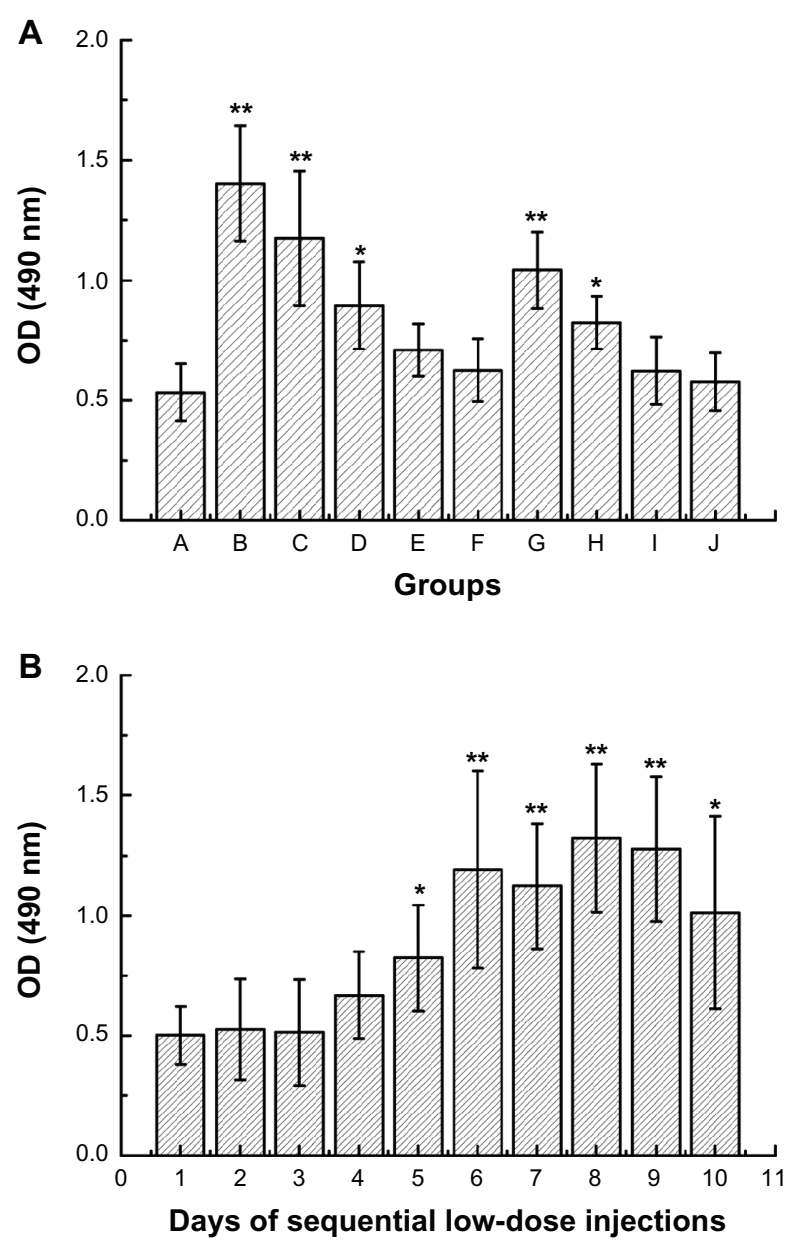

Figure 3 The production of anti-PEG IgM in rats. (A) Groups A to J represent predosing with $5 \%$ glucose injection; I, 5, 10, 15, or $50 \mu \mathrm{mol}$ phospholipids $/ \mathrm{kg}$ empty liposomes; and I, 5, I0, or I5 $\mu \mathrm{mol}$ phospholipids $/ \mathrm{kg}$ epirubicin liposomes. Anti-PEG IgM in the serum was determined at the seventh day after the first administration and before the last day of repeated injections (B).

Notes: Data are shown as mean $\pm S D, n=3$. P-values apply to difference between the control and treated rats. $* P<0.05$; $* * P<0.01$.

Abbreviation: IgM, immunoglobulin M; OD, optical density; PEG, polyethylene glycol; SD, standard deviation.

the amounts of anti-PEG IgM with other groups (groups B-J) were all higher than the control group, even if in groups $\mathrm{F}$ and $\mathrm{J}$ the increased blood clearance of the second injection of liposomes had completely disappeared. The above results indicated that the induction of the $\mathrm{ABC}$ phenomenon was dose-dependent and required a minimum starting dose. From the results of Figure 3B, we found that 5 days of repeated low-dose chemotherapy with $0.75 \mathrm{mg} / \mathrm{kg}$ EPI encapsulated PEGylated liposomes had already induced the production of anti-PEG IgM, and it reached a plateau after 6 to 10 days of repeated injections.

\section{EPI released from liposomes}

To further illustrate the changes in the pharmacokinetic behavior of the drug itself during the $\mathrm{ABC}$ phenomenon, 


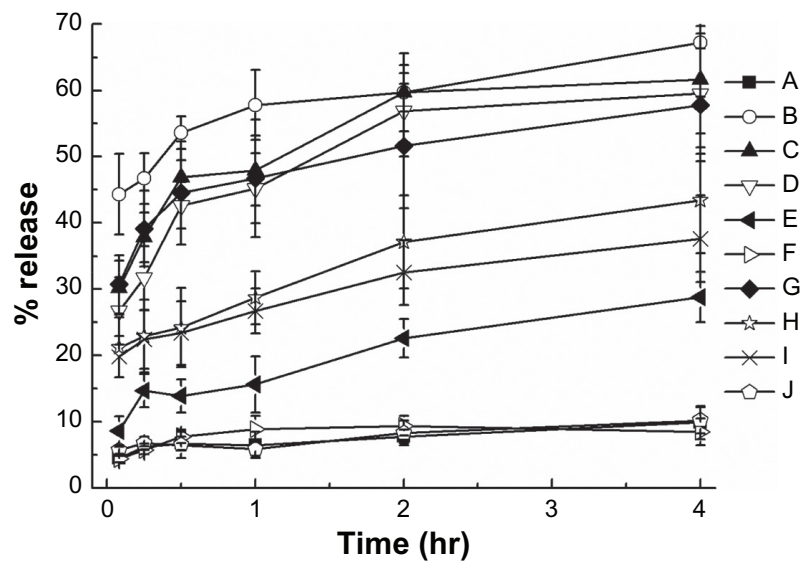

Figure 4 The leakage of epirubicin from liposomes during incubation with the rat sera. Notes: Groups A to J represent the sera which were collected from rats that had already been pre-dosed with a $5 \%$ glucose injection; I, 5, 10, 15, or $50 \mu \mathrm{mol}$ phospholipids/kg empty liposomes; and I, 5, 10, or $15 \mu \mathrm{mol}$ phospholipids $/ \mathrm{kg}$ epirubicin liposomes respectively. Data are shown as mean $\pm S D, n=3$. Abbreviation: SD, standard deviation.

we incubated EPI encapsulated liposomes with untreated rat serum and serum obtained from the rats 1 week after the first injection. The EE of EPI at different times was used as a measure to determine the degree of EPI released from the liposomes. Figure 4 shows the results of the release rate when liposomes were mixed with serum. For the control group (group A) and groups $\mathrm{F}$ and $\mathrm{J}$, almost no drug release was observed during the 4 hours of incubation. Approximately $15 \%$ of the EPI released from the liposomes after 30 minutes of incubation for groups E, H, and I. Additionally, a rapid decrease in entrapment efficiency of EPI liposomes accompanying more than $40 \%$ drug release was determined in groups $\mathrm{B}, \mathrm{C}, \mathrm{D}$, and $\mathrm{G}$. Comparing the results of Figure 1A and Figure 4, we found that the clearance of drug in vivo corresponded to its in vitro release rate. Similar results of accelerated EPI release were also observed with repeated 5 to 10 days of sequential low-dose injections (data not shown). This is consistent with our previous forecast and may also explain why the fast elimination of EPI did not result in a significantly increased liposome uptake in the liver and spleen.

\section{Residual complement activity in serum pre-dosed with PEGylated liposomes}

Complement systems play an important role in the opsonization of PEGylated liposomes and may also promote the leakage of encapsulated drugs when they are activated by the complex of PEGylated liposomes and the antibody of anti-PEG IgM. To explain the phenomenon, the residual complement activity in serum was determined. As indicated in Figure 5A,
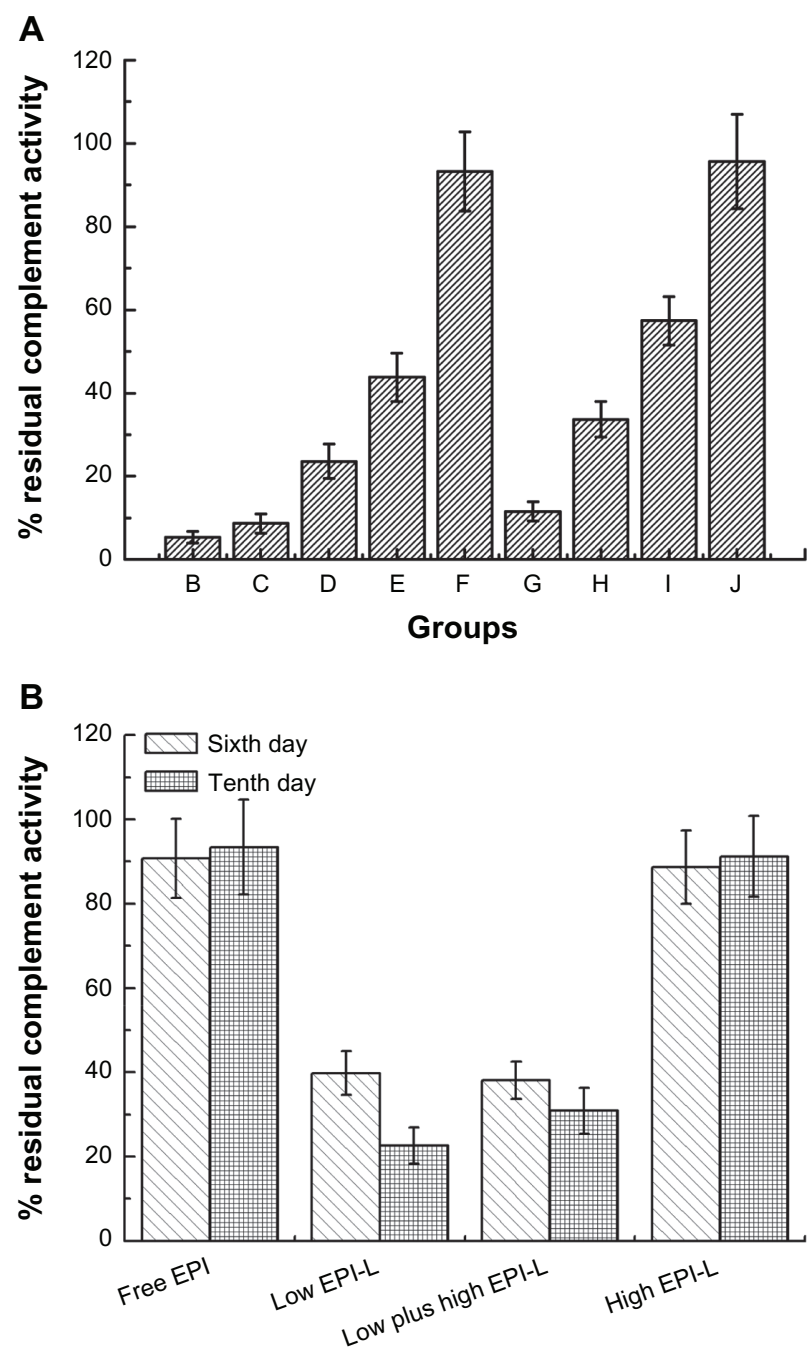

Figure 5 The residual complement activity in rats and mice serum. (A) Groups B to J represent pre-dosing with I, 5, 10, 15, or $50 \mu \mathrm{mol}$ phospholipids $/ \mathrm{kg}$ empty liposomes and I, 5, I0, or I5 $\mathrm{mmol}$ phospholipids $/ \mathrm{kg}$ epirubicin liposomes. (B) The residual complement activity in mice serum.

Notes: Mice were injected via the tail vein with EPI dose of $0.75 \mathrm{mg}$ liposomal $\mathrm{EPI} / \mathrm{kg}$ (Low EPI-L), $1.5 \mathrm{mg}$ liposomal EPI $/ \mathrm{kg}$ (High EPI-L), or $0.75 \mathrm{mg}$ free EPI $/ \mathrm{kg}$ (Free-EPI), once daily for 10 days. In addition, the group of Low plus high EPI-L was injected with $0.75 \mathrm{mg}$ liposomal EPI/ $\mathrm{kg}$ for the first 5 days, and then the dose was increased to $1.5 \mathrm{mg}$ liposomal $\mathrm{EPI} / \mathrm{kg}$. Data are shown as mean $\pm \mathrm{SD}, \mathrm{n}=3$ for rats and $\mathrm{n}=5$ for mice.

Abbreviations: EPI, epirubicin; SD, standard deviation.

a considerable portion of the complement was consumed when incubated with PEGylated liposomes in a drug and phospholipids dose-dependent way; the less the injected dose, the greater complement activation. Figure 5B represents the residual complement activity in mice serum after sequential injections. For free-EPI and High EPI-L groups, sequential injections after 6 and 10 days almost did not alter the residual complement activity. However, the residual complement activity was less than $40 \%$ with Low EPI-L and Low plus high EPI-L groups on the sixth day, and it was further reduced to about $20 \%$ after 10 days of sequential injections. 

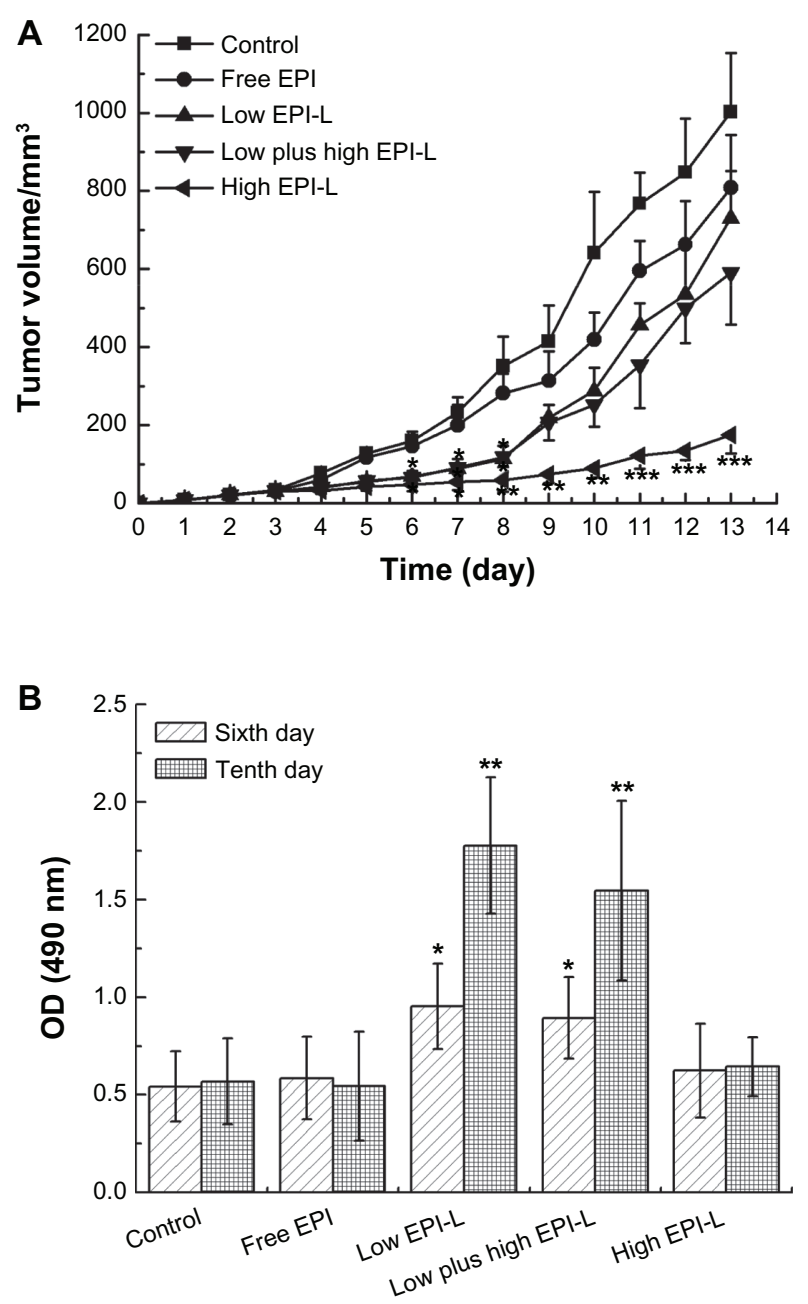

Figure 6 The anti-tumor activity of sequential low-dose injected epirubicin liposomes on SI80 tumor-bearing mice. (A) Tumor volume. (B) The determination of anti-PEG IgM following repeated injections of $5 \%$ glucose, free epirubicin, and epirubicin-encapsulated PEGylated liposomes.

Notes: SI 80 tumor-bearing mice were injected via the tail vein with EPI dose of 0.75 $\mathrm{mg}$ liposomal EPI/kg (Low EPI-L), I.5 mg liposomal EPI/kg (High EPI-L), or 0.75 mg free $\mathrm{EPI} / \mathrm{kg}$ (Free EPI), or the same volumes of $5 \%$ glucose (Control), once daily for 10 days. In addition, the group of Low plus high EPI-L was injected with $0.75 \mathrm{mg}$ liposomal EPI/ $\mathrm{kg}$ for the first 5 days, and then the dose was increased to $1.5 \mathrm{mg}$ liposomal EPI/ $\mathrm{kg}$. Data are shown as mean $\pm S D, n=5-10$. P-values apply to differences between the free epirubicin and liposome-treated rats. $* P<0.05$; $* * P<0.0$ I; $* * * P<0.00$ I. Abbreviations: $\mathrm{EPI}$, epirubicin; $\mathrm{OD}$, optical density; SD, standard deviation.

\section{Therapeutic efficacy of sequential low- dose injections of EPI liposomes on SI 80 tumor-bearing mice}

S180 tumor-bearing mice were used to investigate the antitumor activity of EPI liposomes following sequential lowdose injections. As shown in Figure 6A, compared with the control group, the free EPI had almost no anti-tumor effects, and sequential injection of EPI encapsulated liposomes with a dose of $1.5 \mathrm{mg}$ EPI $/ \mathrm{kg}$ significantly inhibited tumor growth. For the other groups, the rapid increase in tumor volume was inhibited to a certain degree within the first 5 days after administration. However, the tumor rapidly grew following the sixth to tenth days even though the dose of EPI liposomes was doubled for the Low plus high EPI-L treated mice. The results demonstrated that the first day to the fifth day injections of low-dose PEGylated EPI liposomes may have already activated the immune system and that resulted in the rapid release and clearance of high-dose PEGylated EPI liposomes that were injected during the final 5 days.

Figure $6 \mathrm{~B}$ shows the amounts of anti-PEG IgM after repeated injections. For the Low EPI-L and Low plus high EPI-L groups, compared with the sixth day, the level of antiPEG IgM in mice on the tenth day after injection significantly increased. No such differences were seen with the control, free-EPI, and High EPI-L groups. The adsorption of the produced anti-PEG IgM on the surface of the subsequently injected liposomes may have induced the leakage of EPI from the liposomes. It may be the reason why the tumor volume rebounded.

\section{The tissue distribution of sequentially injected EPI liposomes in SI 80 tumor- bearing mice}

The emergence of anti-PEG IgM during repeated low-dose chemotherapy indirectly adversely affects the anti-tumor efficacy of subsequently administrated liposomes, and the direct reason for the reduction in therapeutic efficacy may be the alteration of biodistribution upon sequential low-dose injections. Therefore, the tissue distributions of injected EPI liposomes were determined. Figure 7A and B represent the biodistributions of EPI on the sixth and tenth day after injection, respectively. For Low EPI-L and Low plus high EPI-L groups, both on the sixth and tenth day, the concentrations of EPI in the liver and spleen sharply increased, accompanied by reduced amounts of EPI in the tumor. However, for the High EPI-L group, EPI concentrations in blood and tumor were always higher than other tissues whether it was on the sixth or tenth day, and at the same time, no significant hepatic and splenic accumulation was detected.

\section{Discussion}

The method of active or remote loading of drugs into liposomes has been rapidly developed in recent years. ${ }^{32}$ The establishment of an ion gradient is one of the key factors involved in an effective improvement in the entrapment efficacy. In our study, ion exchange fiber technology was used to create a gradient of ammonium sulfate in liposomes. Compared with the conventional gel filtration technology, this method removed ammonium sulfate in the aqueous 

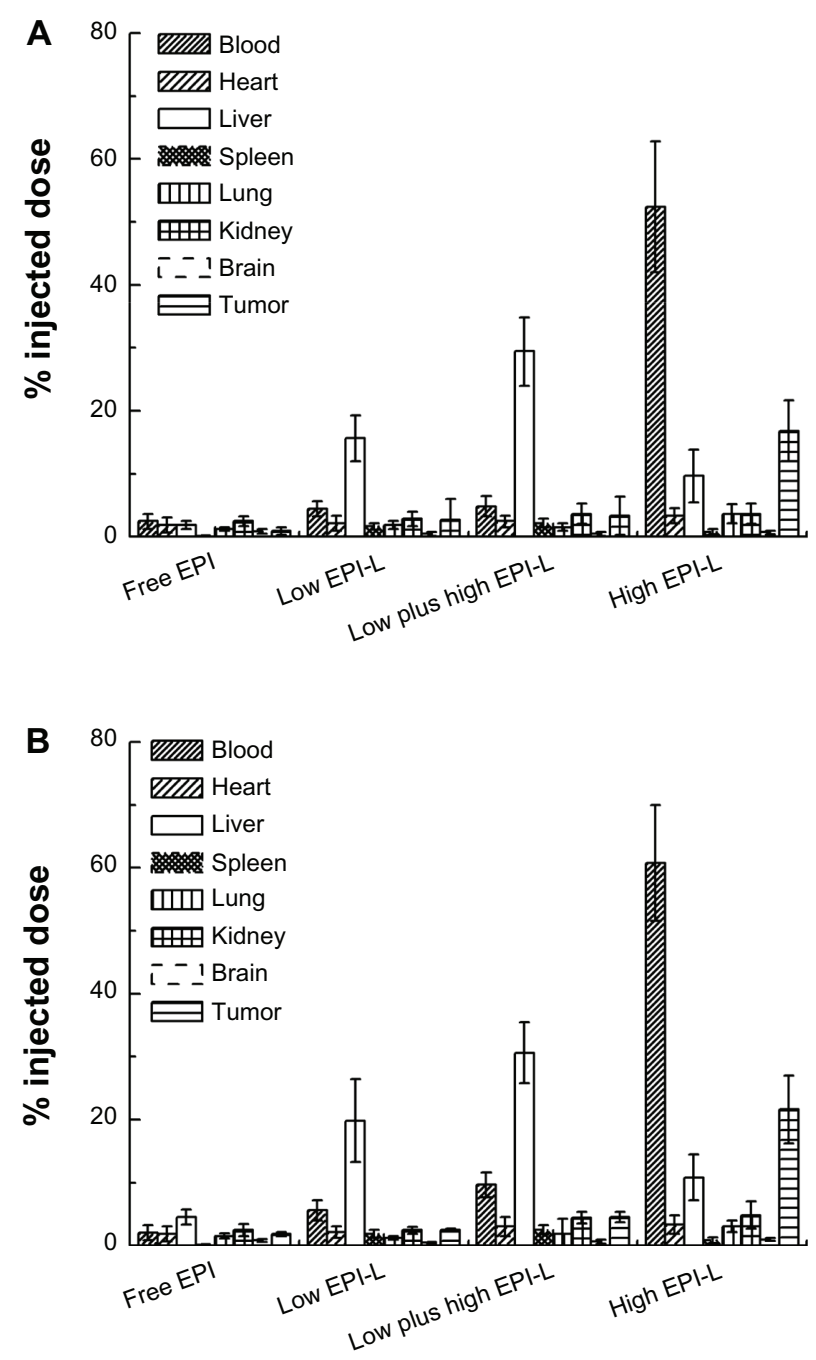

Figure 7 The tissue distributions of sequentially low-dose injected epirubicin liposomes in SI80 tumor-bearing mice. (A) The concentrations of epirubicin in the blood and tissue of mice on the sixth day, 4 hours after injection. (B) The concentrations of epirubicin in the blood and tissue of mice on the tenth day, 4 hours after injection.

Notes: Mice were injected via the tail vein with EPI dose of $0.75 \mathrm{mg}$ liposomal EPI $/ \mathrm{kg}$ (Low EPI-L), I.5 mg liposomal EPI/kg (High EPI-L), or $0.75 \mathrm{mg}$ free EPI/kg (Free-EPI), once daily for 10 days. In addition, the group of Low plus high EPI-L was injected with $0.75 \mathrm{mg}$ liposomal EPI $/ \mathrm{kg}$ for the first 5 days, and then the dose was increased to $1.5 \mathrm{mg}$ liposomal EPI/ $\mathrm{kg}$. Data are shown as mean $\pm \mathrm{SD}, \mathrm{n}=5$.

Abbreviations: EPI, epirubicin; SD, standard deviation.

exterior of the liposomes rapidly and encapsulated drugs into the liposomes effectively. Moreover, this method does not destroy the stability of the liposomes and is inexpensive.

Early reports have shown that the time interval between injections significantly affected the extent of blood clearance and hepatic accumulation of the second injection of PEGylated liposomes. Ishida et $\mathrm{al}^{33}$ showed that at days 4 to 6 post-first injection, clearly increased blood clearance was observed in rats. Combined with our preliminary experiments, 7 days was selected as the time interval to induce the $A B C$ phenomenon.
Subsequent analysis showed that the main differences between our study and the earlier report ${ }^{11}$ were that the liposomes were respectively labeled with EPI and ${ }^{3} \mathrm{H}-\mathrm{CHE}$. During the $\mathrm{ABC}$ phenomenon, if leakage of EPI from the second injection of liposomes occurred, the contradiction was easy to explain. To investigate the in vivo clearance of liposomes, the fluorescent phospholipid of NBD-PE was used to label the carrier, since its higher detection sensitivity and wider linear range were convenient to monitor the pharmacokinetic change of the liposomes when the $\mathrm{ABC}$ phenomenon existed. Meanwhile we investigated the in vivo behavior of the liposomes, and the results indicated that the clearance of the carrier (liposome) was slower than the drug (EPI).

To discuss the effect of anti-PEG IgM secreted by the $\mathrm{B}$ cells in the splenic marginal zone on the release of subsequently administrated liposomal EPI, liposomes were incubated with sera and the release rate of EPI was determined. The rapid release of EPI was observed when liposomes were incubated with serum containing anti-PEG IgM. This was mainly due to the activation of the complement system following anti-PEG IgM binding to the surface of the liposomes. There are two factors involved in the complement system of defense against invading (second or sequential injected) liposomes. The major one is to remove the liposomes by the phagocytic cells through the opsonization of activated C3 fragments. ${ }^{22}$ This plays a critical role in the clearance of liposomes during the $\mathrm{ABC}$ phenomenon. Another important role of the complement system to antagonize the subsequently injected liposomes is the formation of a membrane attack complex by the assembly of a C5b-9 complex. ${ }^{23}$ The membrane attack complex is inserted into the bilayer of liposomes and pores with a diameter of $10 \mathrm{~nm}$ are produced ${ }^{34}$ which, in turn, induces the release of drugs encapsulated in the liposomes. Interestingly, the rapid release of EPI occurred during the first 30 minutes, which was very similar to the enhanced blood clearance behavior of the second injection of EPI-labeled liposomes in vivo. This may be the reason why the activation of the immune system by subsequently injected liposomes was almost not time-dependent and a considerable amount of anti-PEG IgM was consumed during the early stages of the immune response. The determination of residual complement activity in serum that was pretreated with PEGylated liposomes also demonstrated that a massive amount of complement was consumed in the early stages of the $\mathrm{ABC}$ phenomenon. Integration of the above results allows us to speculate that the $\mathrm{ABC}$ phenomenon is simultaneously accompanied by enhanced clearance of the carriers and rapid 
drug leakage. This means that the pharmacokinetics and biodistribution of the drug will inevitably be different from that of the carrier (ie, empty PEGylated liposomes).

The production of marked therapeutic efficacy is the main reason for encapsulating drugs into liposomes, and sequential low-dose injections have the advantage of producing lower side effects and attenuated multidrug resistance. The combination of liposomes and the above method may significantly improve the anti-tumor activity of liposomal cytotoxic drugs. Although repeated low-dose chemotherapy of liposomes has many potential advantages, it may also have the risk of causing the $\mathrm{ABC}$ phenomenon. Therefore, the pharmacokinetics and biodistribution were investigated following sequential low-dose injections of EPI-labeled liposomes in rats. The results in our tests indicated that 5 to 10 days of repeated injections were enough to induce the $\mathrm{ABC}$ phenomenon. The study of the anti-tumor activity of repeated low-dose chemotherapy in mice further illustrated that the treatment efficacy disappeared after the sixth day of repeated injections, accompanied by an increased level of anti-PEG IgM and decreased residual complement activity in mice serum. In addition, no improvement was observed even though the subsequent dose was doubled. Unlike the High EPI-L group, the antibody induced by the sequential injection of low-dose liposomes for 5 days may have resulted in the rapid leakage of EPI from the liposomes together with an increased clearance of the subsequently administered liposomes. Consequently, the concentrations of EPI in tumor tissue declined sharply and this prediction was indeed confirmed by the tissue distribution results. This means that the scheme of sequential low-dose chemotherapy may be inappropriate when PEGylated liposomes are used as carriers, especially when loaded with non-cytotoxic agents.

\section{Conclusion}

In the present study, the $\mathrm{ABC}$ phenomenon and the rapid release and clearance of EPI from the subsequently injected liposomes were induced by repeated injections of lowdose EPI-encapsulated PEGylated liposomes, and this severely affected the therapeutic efficacy of the sequential low-dose injections. Therefore, when administered in a sequential manner, a more reasonable dose and treatment scheme should be selected to reduce or even avoid this phenomenon.

\section{Acknowledgment}

This study was supported by the National Natural Science Foundation of China (Grant No 81072602).

\section{Disclosure}

The authors report no conflicts of interest in this work.

\section{References}

1. Lasic DD. Novel applications of liposomes. Trends Biotechnol. 1998;16(7):307-321.

2. Torchilin VP. Recent advances with liposomes as pharmaceutical carriers. Nat Rev Drug Discov. 2005;4(2):145-160.

3. Allen TM, Hansen C, Martin F, Redemann C, Yau-Young A Liposomes containing synthetic lipid derivatives of poly(ethylene glycol) show prolonged circulation half-lives in vivo. Biochim Biophys Acta. 1991;1066(1):29-36.

4. Klibanov AL, Maruyama K, Torchilin VP, Huang L. Amphipathic polyethyleneglycols effectively prolong the circulation time of liposomes. FEBS Lett. 1990;268(1):235-237.

5. Iyer AK, Khaled G, Fang J, Maeda H. Exploiting the enhanced permeability and retention effect for tumor targeting. Drug Discov Today. 2006;11(17-18):812-818.

6. Yokoyama M. Drug targeting with nano-sized carrier systems. J Artif Organs. 2005;8(2):77-84.

7. Dams ET, Laverman P, Oyen WJ, et al. Accelerated Blood Clearance and Altered Biodistribution of Repeated Injections of Sterically Stabilized Liposomes. J Pharmacol Exp Ther. 2000;292(3):1071-1079.

8. Wang XY, Ishida T, Ichihara M, Kiwada H. Influence of the physicochemical properties of liposomes on the accelerated blood clearance phenomenon in rats. J Control Release. 2005;104(1):91-102.

9. Xu H, Wang KQ, Deng YH, Chen DW. Effects of cleavable PEGcholesterol derivatives on the accelerated blood clearance of PEGylated liposomes. Biomaterials. 2010;31(17):4757-4763.

10. Laverman P, Carstens MG, Boerman OC, et al. Factors Affecting the Accelerated Blood Clearance of Polyethylene GlycolLiposomes upon Repeated Injection. J Pharmacol Exp Ther. 2001;298(2):607-612.

11. Ishida T, Atobe K, Wang X, Kiwada H. Accelerated blood clearance of PEGylated liposomes upon repeated injections: Effect of doxorubicinencapsulation and high-dose first injection. $J$ Control Release. 2006;115(3):251-258

12. Ishida T, Kiwada H. Accelerated blood clearance (ABC) phenomenon upon repeated injection of PEGylated liposomes. Int J Pharm. 2008;354(1-2):56-62.

13. Ishida T, Ichikawa T, Ichihara M, Sadzuka Y, Kiwada H. Effect of the physicochemical properties of initially injected liposomes on the clearance of subsequently injected PEGylated liposomes in mice. J Control Release. 2004;95(3):403-412.

14. Ma Y, Yang Q, Wang L, Zhou X, Zhao Y, Deng Y. Repeated injections of PEGylated liposomal topotecan induces accelerated blood clearance phenomenon in rats. Eur J Pharm Sci. 2012;45(5):539-545.

15. Seo JW, Zhang H, Kukis DL, Meares CF, Ferrara KW. A novel method to label preformed liposomes with $64 \mathrm{Cu}$ for positron emission tomography (PET) imaging. Bioconjug Chem. 2008;19(12):2577-2584.

16. Sakaguchi N, Kojima C, Harada A, Koiwai K, Kono K. The correlation between fusion capability and transfection activity in hybrid complexes of lipoplexes and pH-sensitive liposomes. Biomaterials. 2008;29(29): 4029-4036.

17. Almofti MR, Harashima H, Shinohara Y, Almofti A, Baba Y, Kiwada H. Cationic liposome-mediated gene delivery: Biophysical study and mechanism of internalization. Arch Biochem Biophys. 2003;410(2): 246-253.

18. Kerbel RS, Kamen BA. The anti-angiogenic basis of metronomic chemotherapy. Nat Rev Cancer. 2004;4(6):423-436.

19. Zhang L, Yu D, Hicklin DJ, Hannay JAF, Ellis LM, Pollock RE. Combined Anti-Fetal Liver Kinase 1 Monoclonal Antibody and Continuous Low-Dose Doxorubicin Inhibits Angiogenesis and Growth of Human Soft Tissue Sarcoma Xenografts by Induction of Endothelial Cell Apoptosis. Cancer Res. 2002;62(7):2034-2042. 
20. Jeremic B, Shibamoto Y, Stanisavljevic B, Milojevic L, Milicic B, Nikolic N. Radiation therapy alone or with concurrent low-dose daily either cisplatin or carboplatin in locally advanced unresectable squamous cell carcinoma of the head and neck: a prospective randomized trial. Radiother Oncol. 1997;43(1):29-37.

21. Klement G, Baruchel S, Rak J, et al. Continuous low-dose therapy with vinblastine and VEGF receptor-2 antibody induces sustained tumor regression without overt toxicity. J Clin Invest. 2000;105(8):R15-R24.

22. Bradley AJ, Devine DV. The complement system in liposome clearance: Can complement deposition be inhibited? Adv Drug Deliv Rev. 1998;32(1-2):19-29.

23. Podack ER, Stoffel W, Esser AF, Müller-Eberhard HJ. Membrane attack complex of complement: distribution of subunits between the hydrocarbon phase of target membranes and water. Proc Natl Acad Sci U S A. 1981;78(7):4544-4548.

24. Yang Y, Ma Y, Wang S. A novel method to load topotecan into liposomes driven by a transmembrane NH4EDTA gradient. Eur J Pharm Biopharm. 2012;80(2):332-339.

25. Haran G, Cohen R, Bar LK, Barenholz Y. Transmembrane ammonium sulfate gradients in liposomes produce efficient and stable entrapment of amphipathic weak bases. Biochim Biophys Acta. 1993;1151(2): 201-215.

26. Katewa SD, Katyare SS. A simplified method for inorganic phosphate determination and its application for phosphate analysis in enzyme assays. Anal Biochem. 2003;323(2):180-187.

27. Wang X, Ishida T, Kiwada H. Anti-PEG IgM elicited by injection of liposomes is involved in the enhanced blood clearance of a subsequent dose of PEGylated liposomes. J Control Release. 2007;119(2):236-244.
28. Ishida T, Wang X, Shimizu T, Nawata K, Kiwada H. PEGylated liposomes elicit an anti-PEG IgM response in a $\mathrm{T}$ cell-independent manner. J Control Release. 2007;122(3):349-355.

29. Ichihara M, Shimizu T, Imoto A, et al. Anti-PEG IgM Response against PEGylated Liposomes in Mice and Rats. Pharmaceutics. 2011;3(1):1-11.

30. Gabizon A, Catane R, Uziely B, et al. Prolonged Circulation Time and Enhanced Accumulation in Malignant Exudates of Doxorubicin Encapsulated in Polyethylene-glycol Coated Liposomes. Cancer Res. 1994;54(4):987-992.

31. Mayhew EG, Lasic D, Babbar S, Martin FJ. Pharmacokinetics and antitumor activity of epirubicin encapsulated in long-circulating liposomes incorporating a polyethylene glycol-derivatized phospholipid. Int J Cancer. 1992;51(2):302-309.

32. Haran G, Cohen R, Bar LK, Barenholz Y. Transmembrane ammonium sulfate gradients in liposomes produce efficient and stable entrapment of amphipathic weak bases. Biochim Biophys Acta. 1993;1151(2):201-215.

33. Ishida T, Ichihara M, Wang X, et al. Injection of PEGylated liposomes in rats elicits PEG-specific IgM, which is responsible for rapid elimination of a second dose of PEGylated liposomes. J Control Release. 2006;112(1):15-25.

34. Muller-Eberhard HJ. The membrane attack complex of complement. Annu Rev Immunol. 1986;4:503-528.
International Journal of Nanomedicine

\section{Publish your work in this journal}

The International Journal of Nanomedicine is an international, peerreviewed journal focusing on the application of nanotechnology in diagnostics, therapeutics, and drug delivery systems throughout the biomedical field. This journal is indexed on PubMed Central, MedLine, CAS, SciSearch $\AA$, Current Contents ${ }^{\circledR} /$ Clinical Medicine,

\section{Dovepress}

Journal Citation Reports/Science Edition, EMBase, Scopus and the Elsevier Bibliographic databases. The manuscript management system is completely online and includes a very quick and fair peer-review system, which is all easy to use. Visit http://www.dovepress.com/ testimonials.php to read real quotes from published authors. 